\title{
Development of a Sievert's type gas doping apparatus
}

\author{
B BANDYOPADHYAY, A GHOSHRAY and N CHATTERJEE \\ Saha Institute of Nuclear Physics, Calcutta 700 009, India \\ MS received 14 April 1987
}

\begin{abstract}
A high pressure and high temperature Sicvert's type gas doping apparatus using indigenous components has been designed and fabricated for preparing metal hydrides. This has been tested by preparing vanadium hydride $\mathrm{VH}_{0.8}$. Proton NMR of this hydride has been observed and compared with earlier reports.
\end{abstract}

Keywords. Metal hydrides; hydriding apparatus.

\section{Introduction}

Recently, research on hydrogen in metals has attracted considerable attention both from theoretical as well as applied points of view. The electronic and magnetic properties of hydrides of some transition metals and their intermetallic compounds are being studied. These materials are normally hydrided at elevated temperatures in the presence of hydrogen at a high pressure and the hydriding reaction is therefore done in a closed chamber called the hydriding apparatus. We have designed and fabricated a Sievert's type hydriding apparatus (Mueller et al 1968) using indigenous components and the hydriding experiment has been successfully carried out in the apparatus.

The working range of temperature and pressure for the hydriding apparatus to be fabricated has to be suitably selected for the particular systems of interest. The phase diagrams of different hydrides provide the appropriate temperatures and pressures for their synthesis. In our case, however, the availability of components as well as the ease of fabrication also had to be considered and finally an apparatus was set up where hydriding experiments at a pressure of upto 3.5 MPa and in the temperature range from room temperature to $700^{\circ} \mathrm{C}$ were carried out.

\section{Development}

Figure 1' shows the details of the apparatus consisting of four main parts, viz the purifiers, the reservoir, the reactor chamber and the manometer with a standard volume. Different parts were connected by stainless steel tubes (i.d. $=4 \mathrm{~mm}$ ) using compression type fittings. The inner volume of the tubes was reduced by inserting ceramic capillaries throughout the length. Only needle-type valves were used in our system. The valves were thoroughly checked before connecting to the system. While working with hydrogen, the complete gas-line manifold has to be absolutely leakproof and leak tests should be conducted from time to time. The whole system was tested for leak-proof at $5 \mathrm{MPa}$. Also a vacuum of the order of $10^{-6}$ torr could he achieved and maintained throughout the manifold. 


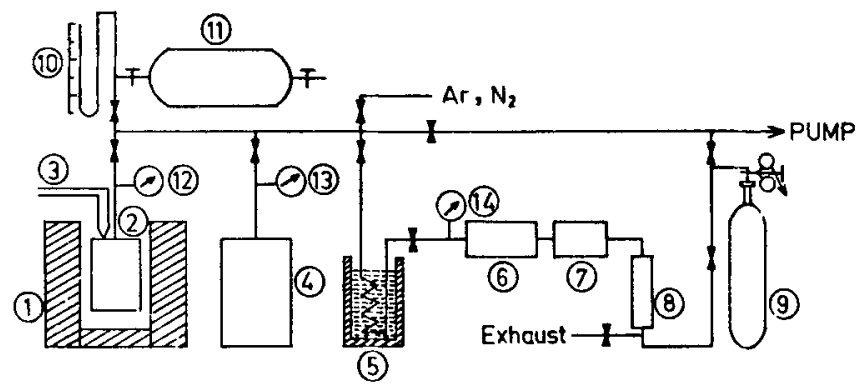

Figure 1. The hydriding apparatus. (1) furnace, (2) reactor chamber, (3) thermo-couple. (4) reservoir, (5) liquid $\mathrm{N}_{2}$ trap, (6) activated charcoal container, (7) $\mathrm{KOH}$ pellet container, (8) silica gel column, (9) $\mathrm{H}_{2}$ cylinder, (10) $\mathrm{Hg}$ manometer with scale, (11) standard volume and (12-14) pressure gauges.

\subsection{Purifier}

The hydrogen gas (IOLAR-2), supplied by the Indian Oxygen Limited, contains various impurities such as nitrogen (100 VPM), oxygen (4 VPM), moisture (4 VPM), oxides of carbon and nitrogen (2 VPM) and trace amounts of hydrocarbon and sulphur components. These impurities often exert an undesirable influence on the preparation of hydrides. For example, nitrogen and oxygen may create films of nitride and oxide respectively on the material surface preventing diffusion of hydrogen into the bulk. Some hydrides tend to lose their hydrogen concentration in the presence of moisture. Other impurities, in general, may adversely affect the hydriding process by surface poisoning.

To remove the impurities, hydrogen from the cylinder was made to pass slowly through a train of purifiers before reaching the reactor chamber. Moisture was removed by using a column of dehydrated silica gel. Potassium hydroxide pellets were packed in a horizontal column to remove carbon-dioxide through the reaction, $\mathrm{KOH}+\mathrm{CO}_{2} \rightarrow \mathrm{KHCO}_{3}$. Powdered activated charcoal was used as nitrogen getter. Finally, heavier gaseous impurities were arrested in a liquid nitrogen trap.

\subsection{Reservoir}

Purified and measured quantities of hydrogen can be stored here, if necessary. Pressure of the gas is measured using a Bourdon-type gauge.

\subsection{Reactor chamber}

Figure 2 illustrates the reactor chamber suitably designed to run for a number of heating and cooling cycles at high temperatures and pressures in an absolutely leakproof condition. The whole chamber was cut out from a $62 \mathrm{~mm}$ rod of stainless steel which is the best chosen material for the reactor because of its hardness, small thermal expansion coefficient, low porosity and low gas absorption coefficient. Flange-type sealing with a metal gasket was. used. Flat copper gasket, earlier annealed in hydrogen atmosphere and polished, was placed between the two circular 


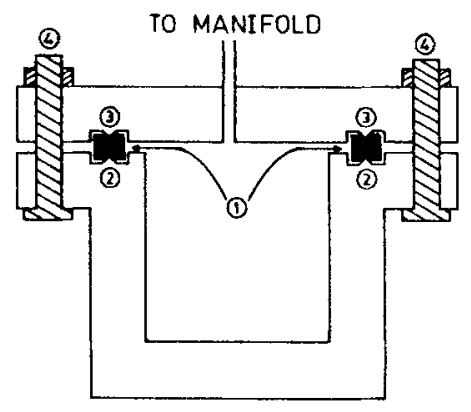

Figure 2. Cross-sectional view of the reactor chamber. (1) $\mathrm{Cu}$ gasket, (2 and 3) ndges on upper and lower flanges and (4) nuts and bolts.

ridges of the upper and the lower flanges. Of the various types of sealing tried, this was felt the most suitable method of sealing the reactor to work at a high pressure and a temperature as high as $700^{\circ} \mathrm{C}$. Stainless steel bolts and nuts were used to maintain equal thermal expansion. The reactor chamber was also connected to a Bourdon-type pressure gauge.

\subsection{Manometer}

Concentration of hydrogen in the hydride samples was measured from the amount of gas evolved by thermally degassing a portion of the hydride sample in the reactor chamber and then by using PVT relations. The pressure of the evolved gas was measured from the mercury manometer. The volumes of the reactor chamber and the manometer were accurately measured by PV relations with the help of the manometer and a standard volume which is a cylindrical glass chamber with stop-cocks at both ends. The manometer and the standard volume were connected to the reactor chamber through capillary tubes of copper. The volume of the reservoir was $95 \mathrm{cc}$ and that of the reactor chamber was $28.6 \mathrm{cc}$. The standard volume was $133.7 \mathrm{cc}$ and the manometer had a volume of $8.2 \mathrm{cc}$.

\section{Experiment}

The gas doping apparatus which was fabricated was tested by hydriding pure vanadium. About $1 \mathrm{~g}$ of vanadium in pressed pellet form was etched in a solution of dilute hydrochloric acid and ethyl alcohol. After proper washing and drying, the pellet was taken in the reactor chamber, which was then connected to the manifold. The reactor chamber was then evacuated and flushed several times with hydrogen. The sample was completely outgassed and annealed by holding it for several hours in a dynamic vacuum at $500^{\circ} \mathrm{C}$. Just after degassing, pure hydrogen was slowly injected into the reactor chamber from the reservoir. The rate of increment of pressure inside the reactor chamber, due to hydrogen injection, was less than $5 \mathrm{~mm} / \mathrm{min}$, as observed in the manometer. At this stage, the furnace temperature was lowered down to $450^{\circ} \mathrm{C}$ and a desired amount of hydrogen gas was loaded in the reactor. The reactor was then slowly cooled down to room temperature. 


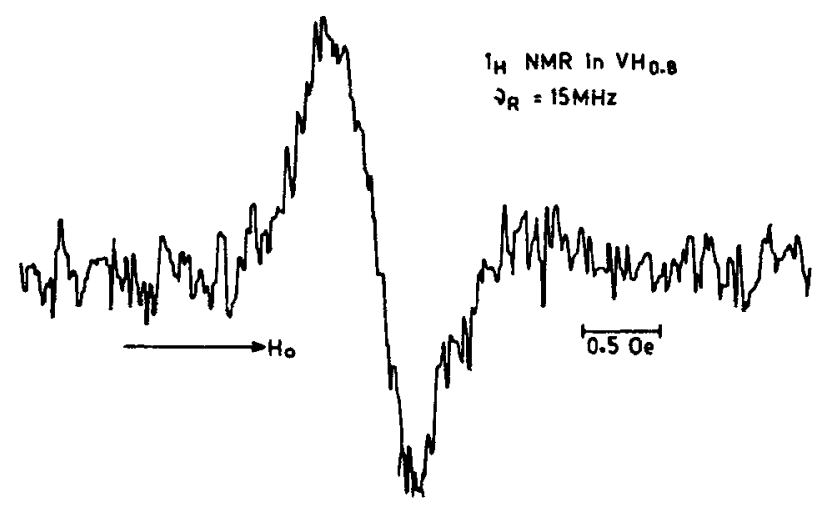

Figure 3. Absorption derivative of ${ }^{1} \mathrm{H}$ NMR in $\mathrm{VH}_{0 \cdot 8}$ at room temperature.

The sample was again completely outgassed at $500^{\circ} \mathrm{C}$ and loaded with hydrogen in the same manner as described above. This method of hydriding and dehydriding the sample was repeated five to six times to activate the metal and finally the same procedure was followed to obtain the desired metal-to-hydrogen ratio in the hydride of vanadium. The reactor was then disconnected from the manifold, cooled in liquid nitrogen and the hydride was taken out and stored in normal atmosphere.

The vanadium hydride, $\mathrm{VH}_{x}$, thus prepared, was then ready for measurements. The value of $x$, the molar hydrogen concentration was measured several times, each time by completely degassing about $0.1 \mathrm{~g}$ of hydride at $500^{\circ} \mathrm{C}$ in the previously evacuated reactor chamber. The pressure developed due to hydrogen liberated from the hydride was observed in the manometer. Using PVT relation, the value of $x$ was calculated as $0 \cdot 8 \pm 0 \cdot 1$.

Proton NMR line of the above $\mathrm{VH}_{0.8}$ was observed in a cw wideline NMR spectrometer using proton-free probe. The line width at room temperature was very narrow, about 0.5 gauss (figure 3), as reported earlier (Zamir and Cotts 1964) for similar hydrides. Thus our fabricated gas-line manifold was tested for successful loading of hydrogen gas under high temperature and high pressure in a properly prepared host metal lattice.

\section{Acknowledgement}

The authors thank Dr K Ghoshray, Mr N Banerjee, Mr D R Sikdar and Mr A Biswas for their advice and active participation in fabricating the apparatus.

\section{References}

Mueller W M, Blackledge J P and Libowitz G G 1968 Metal hydrides (New York and London: Academic Press) p. 151

Zamir D and Cotts R M 1964 Phys. Rev. 134 A666 